\title{
La lealtad de los clientes y su relación con la lealtad de los colaboradores
}

\section{Loyalty of the clients and their relationship with the loyalty of the collaborators}

María Auxiliadora Guerrero Bejarano, MBA

Universidad Internacional del Ecuador, Ecuador

Daniel Ricardo Silva Siu, Msc

Universidad ESAN, Perú

Autor para correspondencia: maguerrerobe@uide.edu.ec, dsilva@pobox.com

Fecha de recepción08 de Junio de 2017 - Fecha de aceptación: 15 de Septiembre de 2017

\section{Resumen}

Muchos académicos y profesionales discuten sobre las diferencias que se presentan en los resultados de las empresas de servicio, desde hace décadas se analiza por qué las organizaciones pierden clientes, aun cuando los gerentes entienden la importancia de estos para la generación de utilidades o mejores resultados, pero la realidad es que en la época actual cada vez es más difícil mantener a los clientes y mucho más fidelizarlo, este trabajo busca analizar la relación que podría existir entre la lealtad de los trabajadores y la lealtad de los clientes en las empresas de servicio.

Palabras Clave: lealtad de trabajadores; lealtad de los clientes; resultados

\begin{abstract}
Many academics and practitioners discuss the differences in service companies' results. For decades, it has been analyzed why organizations lose clients, even though managers understand the importance of these for the generation of profits or better results, but the reality is that in today's era it is increasingly difficult to keep customers and much more loyal, this work seeks to analyze the relationship that could exist between worker loyalty and customer loyalty in service companies.
\end{abstract}

Key words: loyalty of workers; loyalty of clients; results 


\section{Introducción}

\section{Lealtad de los clientes}

De acuerdo con los académicos, los clientes que se consideran habituales (aquellos que suelen repetir sus experiencias en un mismo servicio) son los responsables de generar mayores rendimientos para las empresas, por lo que se afirma que mantener o rentabilizar la relación con un cliente conocido es más rentable que captar un cliente nuevo; pero en la realidad, en los mercados emergentes, a pesar de esta realidad cada vez reuslta más dificil mantener la lealtad de los clientes, entonces es cuando los gerentes de las organizaciones empiezan a cuestionarse ¿por qué los clientes abandonan el servicio? . Es importante empezar por entender qué significa lealtad y los tipos de clientes de acuerdo con la rentabilidad que éstos representan para las organizaciones (Lovelock, Carrion, Huete, Reynoso, \& D'Andrea, 2004).

La lealtad ha sido considerada como algo más que una larga relación de un cliente con una marca determinada o la frecuecia con la que un cliente visita o compra en una tienda, es más bien considerada como la sensación de afinidad que los clientes sienten con los productos o servicios de esta marca (Day, 2000; Hartmann, Apaolaza Ibáñez, \& Forcada Sainz, 2002). Se reconocen distintos tipos de lealtad: (a) la primera fidelidad, que se produce cuando existen una correspondencia favorable entre la actitud del individuo frente a la empresa y su comportamiento de compra, esta representa la situación ideal para la compañía y para el cliente, en estos casos generalmente los clientes suelen actuar como embajadores de la marca; (b) fidelidad latente, el cliente tiene una actitud positiva frente a la marca, pero no manifiesta una lealtad realmente significativa, conocidos como clientes mercenarios, suelen ser clientes que tienen opiniones favorables de la empresa pero no suelen tener comportamiento estable de compra por factores externos, generalmente económicos; (c) fidelidad espúrea, en este tipo el cliente presenta un alto comportamiento de compra, pero generalmente tiene malas opiniones de la marca, generalmente es un cliente rehén, es decir que no tiene la opción de cambiar de proveedor de su servicio; (d) No fidelidad, en este caso, no existe ni actitud, ni comportamiento de compra del cliente hacia la marca, llamados clientes terroristas, suelen darle mala publicidad a la marca (Dick \& Basu, 1994).

\section{Lealtad de los colaboradores}

Se entiende la importancia del personal o cliente interno en una empresa de servicio, cada vez más las empresas se preocupan por conocer qué motiva a sus colaboradores y de qué manera pueden obtener mejores niveles de satisfacción en búsqueda de mejores resultados (Barroso Castro, Martín Armario, \& Martín Ruiz, 2004; Bowers, Martin, \& Luker, 1990; Piercy, Lane, \& Cravens, 2002; Rust, Stewart, Miller, \& Pielack, 1996). Se encuentran pasos o procesos que las organizaciones de servicios deben tener en consideración para buscar el personal de contacto más idóneo para su organización: (a) inversiones en reclutamiento: la mayoría de los costes de contratación son obvios; tasas de reclutamiento, costes de las entrevistas, gastos de recolocación, etc. Sin embargo se debe considerar que contratar a tres aprendices para terminar con un solo empleado productivo a largo plazo - lo cual es lo habitual en las corredurías, los seguros de vida y otras varias industrias -, la verdadera inversión por empleado se triplica. (b) formación, es importante dar a las nuevas incorporaciones una base para el trabajo productivo, con frecuencia significa preparación formal en una clase y también prácticas en el lugar de trabajo. El salario 
que se paga durante el período de formación produce poca o ninguna contribución a la compañía. Aunque las buenas compañías continúen invirtiendo en formación, incluso para su gente más experta, el gasto queda más que compensado por la formación gratuita que los empleados más veteranos dan a sus colegas más jóvenes. En otras palabras, para los empleados leales a largo plazo la formación deja de ser un coste y se convierte en un beneficio neto; (c) eficiencia; en el nivel más simple, los empleados aprenden a trabajar de forma más eficiente a medida que adquieren experiencia en el trabajo. Su eficiencia en aumento significa que requieren menos supervisión, lo cual comporta eficiencias adicionales. Sin embargo, además de estas ventajas, es importante recordar que la eficiencia es el producto de lo inteligentemente que la gente trabaja por multiplicado por lo duro que trabaja. Por lo general, los empleados que permanecen en la compañía porque se enorgullecen del valor que crean para los clientes y porque están complacidos con el valor que crean para sí mismos están más motivados y trabajan más duramente; (d) selección de clientes: los vendedores expertos son mucho mejores para encontrar y reclutar a los mejores clientes. En el negocio de los seguros de vida, por ejemplo, la persistencia en los nuevos negocios es mucho mejor para los agentes expertos que para lo aprendices(Yang \& Peterson, 2004); (e) retención de clientes: los corretajes y los servicios del automóvil, los empleados a largo plazo crean mayor fidelidad de los clientes, sin embargo, incluso en la fabricación, donde los empleados raramente ven a los clientes, los empleados a largo plazo pueden producir productos mejores, mejor valor para el consumidor y mayor retención de clientes (Dewettinck \& van Ameijde, 2011); (f) referencias de los clientes: los empleados leales con frecuencia son una fuente importante de referencias de los clientes, como por ejemplo en los talleres locales; (g) referencias de los empleados: los empleados leales a largo plazo a menudo generan el mejor lujo de solicitantes de empleo de alto calibre. Esto no sólo aumenta la calidad media de los nuevos contratos, sino que también reduce los costes de reclutamiento. Las compañías con los mayores niveles de retención de empleados consecuentemente contratan la gran mayoría de sus nuevas incorporaciones a través de las referencias de los empleados (Dawson, Abbott, \& Shoemaker, 2011; Testa \& Mueller, 2009; Zeithaml, Berry, \& Parasuraman, 1996)

\section{Colaboradores leales, clientes leales}

Muchos académicos afirman que los empleados leales generan clientes leales, pero para conseguir la lealtad de los colaboradores la organización debe estar dispuesta a garantizar el ambiente en el que los clientes internos se desarrollan para conseguir la satisfacción laboral que contribuya a mejores niveles de productividad, lo que desembocaría en la entrega de servicios de valor y al final mejores niveles de satisfacción de los clientes que generaría lealtad y crecimiento de la organización. (Adsit, London, Crom, \& Jones, 1996; Barroso Castro et al., 2004; Bienstock, DeMoranville, \& Smith, 2003; Bowers et al., 1990; Clark, 1997; Yoon \& Suh, 2003)

\section{Conclusiones}

Los clientes responden a la labor de los colaboradores en las empresas de servicio; por esta razón los académicos y profesionales del área se empeñan en sugerir a los gerentes generales de las empresas de las distintas industrias del sector servicio cuidar sus procesos desde el reclutamiento, hasta la forma en que se capacita, bonifica y congratula a los colaboradores de este tipo de empresas, entendiendo que son personas que lidian con sus propias emociones y que deberán atender personas y lidiar con las emociones de estos (Gruber, Abosag, Reppel, \& Szmigin, 2011; Hur, Moon, \& Jung, 2015). 
Los gerentes de las empresas de servicio deben entonces procurar generar un comportamiento de ciudadano organizacional en los colaboradores de sus organizaciones, personas con la intención de dar la milla extra y tener clara la importancia de su labor para la consecución de los objetivos comunes de la organización (Barroso Castro et al., 2004). Existen estudios que han comprobado la relación directa entre la satisfacción de los colaboradores y la de los clientes que permiten obtener mejores resultados para la empresa (Castellanos-Verdugo, de los Ángeles Oviedo-García, Roldán, \& Veerapermal, 2009; José Vilares \& Simões Coelho, 2003) por lo que se podría asegurar que las organizaciones de servicio que obtienen mejores resultados y crecen sosteniblemente lo deben al establecimiento de una cultura y clima organizacional que motiva permanentemente la satisfacción de sus colaboradores en un primer plano para así conseguir la fiabilidad en la prestación de su servicio, entregando propuestas de valor par sus clientes, lo que desembocaría en la lealtad de los mismos (George \& Hegde, 2004; Gruber, 2011; Gruber et al., 2011; Johnston, 1989; Mikic Little \& Dean, 2006; Plymire, 1990).

El rol de la relación colaborador-cliente juega entonces un papel estelar en las empresas denominadas de servicio y serán la fuente de diferenciación entre los competidores, lo que permitiría su crecimiento en el tiempo así como su prolongación de operaciones (Chung-Herrera, Goldschmidt, \& Doug Hoffman, 2004; Edvardsson, 1992; Fisk et al., 2010; Jamal \& Adelowore, 2008)

\section{Bibliografía}

Adsit, D. J., London, M., Crom, S., \& Jones, D. (1996). Relationships between employee attitudes, customer satisfaction and departmental performance. Journal of Management Development, 15(1), 62-75.

Barroso Castro, C., Martín Armario, E., \& Martín Ruiz, D. (2004). The influence of employee organizational citizenship behavior on customer loyalty. International Journal of Service Industry Management, 15(1), 27-53.

Bienstock, C. C., DeMoranville, C. W., \& Smith, R. K. (2003). Organizational citizenship behavior and service quality. Journal of Services Marketing, 17(4), 357-378.

Bowers, M. R., Martin, C. L., \& Luker, A. (1990). Trading places: Employees as customers, customers as employees. Journal of Services Marketing, 4(2), 55-69.

Castellanos-Verdugo, M., de los Ángeles Oviedo-García, M., Roldán, J. L., \& Veerapermal, N. (2009). The employee-customer relationship quality: Antecedents and consequences in the hotel industry. International Journal of Contemporary Hospitality Management, 21(3), 251-274.

Chung-Herrera, B. G., Goldschmidt, N., \& Doug Hoffman, K. (2004). Customer and employee views of critical service incidents. Journal of Services Marketing, 18(4), 241-254.

Clark, M. (1997). Modelling the impact of customer-employee relationships on customer retention rates in a major uk retail bank. Management Decision, 35(4), 293-301.

Dawson, M., Abbott, J., \& Shoemaker, S. (2011). The hospitality culture scale: A measure organizational culture and personal attributes. International Journal of Hospitality Management, 30(2), 290-300.

Day, G. S. (2000). Comprender, captar y fidelizar a los mejores clientes: Grupo Planeta (GBS).

Dewettinck, K., \& van Ameijde, M. (2011). Linking leadership empowerment behaviour to employee attitudes and behavioural intentions: Testing the mediating role of psychological empowerment. Personnel Review, 40(3), 284-305.

Dick, A. S., \& Basu, K. (1994). Customer loyalty: Toward an integrated conceptual framework. Journal of the academy of marketing science, 22(2), 99-113. 
Edvardsson, B. (1992). Service breakdowns: A study of critical incidents in an airline. International Journal of Service Industry Management, 3(4), 17-29.

Fisk, R., Grove, S., Harris, L. C., Keeffe, D. A., Daunt, K. L., Russell-Bennett, R., \& Wirtz, J. (2010). Customers behaving badly: A state of the art review, research agenda and implications for practitioners. Journal of Services Marketing, 24(6), 417-429.

George, B. P., \& Hegde, P. G. (2004). Employee attitude towards customers and customer care challenges in banks. International Journal of Bank Marketing, 22(6), 390-406.

Gruber, T. (2011). I want to believe they really care: How complaining customers want to be treated by frontline employees. Journal of Service Management, 22(1), 85-110.

Gruber, T., Abosag, I., Reppel, A. E., \& Szmigin, I. (2011). Analysing the preferred characteristics of frontline employees dealing with customer complaints: A cross-national kano study. The TQM Journal, 23(2), 128-144.

Hartmann, P., Apaolaza Ibáñez, V., \& Forcada Sainz, F. J. (2002). El efecto del posicionamiento en la lealtad del cliente: Un análisis empírico para el caso de iberdrola.

Hur, W.-M., Moon, T.-W., \& Jung, Y. S. (2015). Customer response to employee emotional labor: The structural relationship between emotional labor, job satisfaction, and customer satisfaction. Journal of Services Marketing, 29(1), 71-80.

Jamal, A., \& Adelowore, A. (2008). Customer-employee relationship: The role of self-employee congruence. European journal of Marketing, 42(11/12), 1316-1345.

Johnston, R. (1989). The customer as employee. International Journal of Operations \& Production Management, 9(5), 15-23.

José Vilares, M., \& Simões Coelho, P. (2003). The employee-customer satisfaction chain in the ecsi model. European journal of Marketing, 37(11/12), 1703-1722.

Lovelock, C. H., Carrion, M. A. S., Huete, L. M., Reynoso, J., \& D'Andrea, G. (2004). Administración de servicios: Pearson Education.

Mikic Little, M., \& Dean, A. M. (2006). Links between service climate, employee commitment and employees' service quality capability. Managing Service Quality: An International Journal, 16(5), 460-476.

Piercy, N. F., Lane, N., \& Cravens, D. W. (2002). A gender perspective on salesperson organizational citizenship behaviour, sales manager control strategy and sales unit effectiveness. Women in Management Review, 17(8), 373-391.

Plymire, J. (1990). How to stop firing your customers. Journal of Services Marketing, 4(2), 49-53.

Rust, R. T., Stewart, G. L., Miller, H., \& Pielack, D. (1996). The satisfaction and retention of frontline employees: A customer satisfaction measurement approach. International Journal of Service Industry Management, 7(5), 62-80.

Testa, M. R., \& Mueller, S. L. (2009). Demographic and cultural predictors of international service worker job satisfaction. Managing Service Quality: An International Journal, 19(2), 195-210.

Yang, Z., \& Peterson, R. T. (2004). Customer perceived value, satisfaction, and loyalty: The role of switching costs. Psychology \& Marketing, 21(10), 799-822.

Yoon, M. H., \& Suh, J. (2003). Organizational citizenship behaviors and service quality as external effectiveness of contact employees. Journal of business research, 56(8), 597-611.

Zeithaml, V. A., Berry, L. L., \& Parasuraman, A. (1996). The behavioral consequences of service quality. the Journal of Marketing, 31-46. 